least-squares solution from 57 velocity measures made at the David Dunlap Observatory since I951. They are as follows: $P=359.7$ days, $\gamma=+7 . \mathrm{I} \mathrm{km} / \mathrm{sec}, K=5 \mathrm{I} .3 \mathrm{~km} / \mathrm{sec}, e=0.27$, $\omega=265^{\circ}, T=\mathrm{JD}_{5} \mathrm{I} 38, a \sin i=2.44 \times \mathrm{IO}^{8} \mathrm{~km}$, $f(m)=4.5 \odot$. The large mass function means that the stars are themselves massive unless there is a very large departure from the massluminosity relation.

Other stars with such large mass functions are eclipsing systems. It is suggested that this star should be examined photometrically at the time of superior conjunction of the primary.

David Dunlap Observatory, Richmond Hill, Ontario.

\section{Hepburn, Nannielou. A photometric study of the solar corona.}

Photometric measurement of the solar corona as photographed by the Naval Research Laboratory expedition to Khartoum, Sudan, on February 25,1952 is described and related to other measurements and theoretical studies. In particular the variation of white light intensity with radius is compared to van de Hulst's model of the corona, and coefficients are derived for his equations which produce curves more nearly fitting the observed intensities. The removal of the $\mathrm{F}$ corona and calculation of electron densities according to van de Hulst's model appears to give consistent results here.

These measurements extend from the solar limb out to about 2.5 solar radii from the center of the sun, so that a combination of the results with those obtained by the French expedition to Khartoum, headed by Bernard Lyot, is of considerable interest. Their photometric and polarimetric measurement of the white light corona extends from about 2.5 solar radii out to more than 20 radii. The variation in brightness with radius as measured here appears to be a direct extension of the far corona as measured by the French both in the equatorial and polar regions. This indicates that the assignment of absolute intensities to these measures by reference to van de Hulst's model is in agreement with the direct absolute intensity measurements. It has also been possible to compare their measurement of the variation with position angle of the red and green coronal lines near the great streamer with variations in the white-light corona. Although the center of the streamer appears to lie about $\mathrm{IO}^{\circ}$ north of the emission line maximum, an examination of the white-light corona near the $\operatorname{limb}(\rho=$ I.I5) shows a very close correlation of white-light intensity with the emission lines.

Naval Research Laboratory,
Washington, D. C.

Herzog, Leonard F. and Pinson, William H., Jr. $\mathrm{Sr}$ and $\mathrm{Rb}$ abundances, elemental and isotopic, and a minimum age for the Homestead meteorite.

The Homestead meteorite is a typical chondrite containing I I per cent metal phase and about 6 per cent sulfide phase. The combined silicate and sulfide phases were extracted and analyzed by isotope dilution for $R b$ and $S r$. Uncontaminated $\mathrm{Sr}$ was extracted by an ion exchange column technique, and its isotopic composition was determined. Precision and accuracy will be discussed.

Duplicate analyses gave the following results: $R b, 4.0 \pm 0.4 \mu \mathrm{gm} / \mathrm{gm} \mathrm{Sr}$, I I.9 \pm 0.4 .

The following table compares the isotopic composition of the Homestead $S r$ to present day Sr.

$\begin{array}{lcccc}\text { Sr isotope abundance } & 84 & 86 & 87 & 88 \\ \text { Homestead } & 0.0057 & 0.0973 & 0.0735 & 0.823\end{array}$ $\begin{array}{lllll}\text { Homestead } & 0.0057 & 0.0973 & 0.0735 & 0.8235 \\ \text { Normal Sr (A. O. Nier) } & 0.0056 & 0.0986 & 0.0702 & 0.8256\end{array}$

The lowest abundance of ${ }^{87} \mathrm{Sr}$ found terrestrially is 0.0690 , in an anorthosite dated geologically as about $2 \times 10^{9}$ years. This suggests that the initial ${ }^{87} \mathrm{Sr}$ terrestrial abundance was slightly lower than this.

Using $\lambda=\mathrm{I} . \mathrm{I} 3 \times \mathrm{IO}^{-11} /$ year for ${ }^{87} R b$, and assuming as the initial abundance 0.0702 , the present terrestrial abundance of ${ }^{87} \mathrm{Sr}$, a minimum age of $3 . \mathrm{I} \pm 0.3 \times \mathrm{IO}^{9}$ years is calculated for the Homestead meteorite. Assuming the reasonable value 0.0690 , the lowest abundance of ${ }^{87} \mathrm{Sr}$ found terrestrially, an age of $4.2 \pm 0.4 \times \mathrm{IO}^{9}$ years is calculated.

The oldest meteorite ages obtained are about $4.6 \times \mathrm{IO}^{9}$ years (Patterson 1953, Wasserberg 1954). Assuming this age for the Homestead meteorite, the initial isotopic abundance of ${ }^{87} \mathrm{Sr}$ was 0.0685 of total $S r$. This figure is of interest in that it again demonstrates the constancy of isotope abundances throughout the solar system in so far as samples are available.

Patterson, C. 1953, Proc. Conf. on Nuclear Processes in Geologic Settings, Williams Bay, Wis., Sept., pp. 36-40. Wasserburg, G. J. and Hayden, R. J. 1955. Phys. Rev., Second Ser., 97, 86.

Department of Geology and Geophysics, Massachusetts Institute of Technology, Cambridge, Mass. 